\section{Comparison of oocyte maturity rates in recombinant Human Chorionic Gonadotropin (HCG) and triptorelin acetate triggers: A prospective randomized study}

\author{
Lakshmanan $\mathbf{S}^{1}$, Saravanan $\mathbf{M}^{1}$, Senthil $\mathbf{P}^{1 *}$ and Sharma $\mathbf{N}^{2}$ \\ ${ }^{1}$ ARC International Fertility Center, 947, Avinashi Road, Puliakulam, Coimbatore-641037, \\ Tamil Nadu, India \\ ${ }^{2}$ Department of Obstetrics and Gynecology, Saveetha University, Kuthambaakam, Chennai \\ 600124, Tamil Nadu, India
}

\section{Abstract}

Luteinizing Hormone (LH) like exposure in the mid cycle for inducing the oocyte maturation is the very crucial step in the success of ICSI treatment. Introduction of LH surge endogenously by $\mathrm{GnRH}$-agonist for final oocyte maturation induction, may be more physiological compared with the administration of HCG. Since GnRH agonist would induce FSH surge also along with LH surge, as happens in natural cycle. However, the effects of giving HCG trigger for inducing only LH surge and giving GnRH agonist trigger for inducing both LH and FSH surge, in patients treated for ICSI with GnRH antagonists need more research. Sub fertile patients planned for ICSI, meeting the requirement of inclusion criteria, were started with recombinant FSH from day 2 of menstrual cycle. GnRH antagonists were started from day 6 of stimulation. FSH dose was adjusted according to the individual response. Trigger was planned when the lead follicle reaches $24 \mathrm{~mm}$. For triggering, 100 patients were randomized to receive Recombinant HCG trigger and Triptorelin acetate trigger. Oocyte retrieval was done 36 hours after Recombinant hCG Trigger and 35 hours after Triptorelin acetate trigger. The oocyte maturity rate was assessed by the number of metaphase II oocytes retrieved.
More Information

*Address for Correspondence: Dr. Priya Senthil, ARC International Fertility Center, 947, Avinashi Road,Puliakulam, Coimbatore- 641037, Tamil Nadu, India, Tel: 8220812314; Email: dr.priyasenthil@hotmail.com; priyasenthil1000@gmail.com

Submitted: 23 September 2020 Approved: 12 October 2020 Published: 13 October 2020

How to cite this article: Lakshmanan $S$ Saravanan M, Senthil P, Sharma N. Comparison of oocyte maturity rates in recombinant Human Chorionic Gonadotropin (HCG) and triptorelin acetate triggers: A prospective randomized study. Clin J Obstet Gynecol. 2020; 3: 123-126.

DOI: 10.29328/journal.cjog.1001064

ORCiD: orcid.org/0000-0002-5631-6480

Copyright: @ 2020 Lakshmanan S, et al. This is an open access article distributed under the Creative Commons Attribution License, which permits unrestricted use, distribution, and reproduction in any medium, provided the original work is properly cited.

Keywords: GnRH agonist trigger; GnRH antagonist cycle; Metaphase 2 oocytes; Oocyte maturation rate; Recombinant hCG Trigger; Triptorelin acetate trigger

Check for updates

OPEN ACCESS

\section{Introduction}

Infertility is the fifth most serious global disability as recognized by the World Health Organization (WHO) and it affects one out of every 6 couples worldwide [1]. Mahmoud Fathalla, the former Director of the WHO Human Reproductive Program quoted "If public health policiers encourage couples to delay and plan pregnancies, then it is equally important that they are assisted in their attempts to conceive in the more limited time available" (Mahmoud Fathalla). Controlled ovarian hyper stimulation, which is a supra physiological process simulating physiological processes that occur during the normal menstrual cycle, like development of follicles, maturation of oocytes. Follicle stimulating Hormone (FSH) and/or Luteinizing Hormone ( $\mathrm{LH}$ ) are started from Day 2 of menstrual cycle to induce the growth of ovarian follicles.
Premature LH surge can occur as follicles grow which could lead to premature ovulation. It can be prevented by the use of a GnRH antagonist in the present cycle [2,3], or continuous administration of a GnRH agonist (GnRHa) to down regulate the GnRH receptor started from the previous cycle [4]. When the follicles reach the optimal size, LH exposure is provided to simulate the mid-cycle LH surge, which induces oocyte maturation and thereby subsequent ovulation [5]. This oocyte retrieval is properly timed following provision of LH exposure to retrieve oocytes. LH exposure initiates the resumption of meiosis and the maturation of the oocyte from the immature "metaphase I" stage to the mature "metaphase II" stage of Oocyte development [6]. During this process of oocyte maturation, the first polar body is extruded such that a diploid cell transitions toward a haploid gamete and attains competence for fertilization by a spermatozoon [6]. 


\section{Materials and method}

Aim

To compare the efficacy of recombinant HCG trigger and Triptorelin acetate trigger in $\mathrm{GnRH}$.

Antagonist, controlled ovarian hyper stimulation cycle.

\section{Study population}

100 patients totally, 50 patients in recombinant HCG trigger group and Triptorelin acetate trigger group.

\section{Inclusion criteria}

Sub fertile patients aged 21-35 years, with body mass index of 18 to $30, \mathrm{AMH} 1.5$ to $4 \mathrm{ng} / \mathrm{dl}$, antral follicular count of 8-24 in both ovaries together and serum estradiol value of 3000 to $5000 \mathrm{pg} / \mathrm{mL}$ are included in the study.

\section{Exclusion criteria}

Sub fertile patients aged more than 35 years with body mass index less 18 and more than 30 , anti mullerian hormone level less than $1.5 \mathrm{ng} / \mathrm{dL}$ and more than $4 \mathrm{ng} / \mathrm{dL}$ and antral follicular count less than 8 and more than 24 in both ovaries together were excluded from the study. And patients with previous IVF failure, $\mathrm{H} / \mathrm{O}$ chemotherapy were also excluded from the study.

\section{Monitoring}

Controlled ovarian hyper stimulation with recombinant FSH was started from day 2 or day 3 of cycle after confirming the pituitary down regulation, i.e. serum estradiol level less than $50 \mathrm{pg} . / \mathrm{mL}$, serum progesterone level less than $0.9 \mathrm{ng} / \mathrm{mL}$, serum Luteinizing hormone level less than $5 \mathrm{IU} / \mathrm{L}$, Endometrial thickness less than $5 \mathrm{~mm}$, No antral follicle larger than $10 \mathrm{~mm}$ in each ovary. Starting dose of Recombinant FSH was based on patient's age, body mass index BMI, antral follicular count and serum anti mullerian hormone level. Inj Cetrorelix 0.25 mg was started subcutaneously from Day 6 of cycle. Follicular growth was assessed by serial transvaginal and serum E2, LH and P4 levels, and Recombinant FSH doses were adjusted accordingly. Ultrasound monitoring of follicular growth was done on day 6 , day 8, day 10 and then daily till trigger. Serum estrogen was measured on day 6, day 8 and day 10 and on the day of trigger. Serum LH was measured on day 6 , day 8 and day 10. And Inj cetrorelix was continued till the day of the trigger. Trigger was planned when the lead follicle size reached $24 \mathrm{~mm}$. For ovulation trigger, the patients with serum estradiol level between 3001-5000 pg./dL were randomly divided into two groups: one group received recombinant HCG $250 \mathrm{mg}$ subcutaneously and the other group received Triptorelin acetate $0.2 \mathrm{mg}$, a GnRH Agonist subcutaneously as ovulation trigger. Both groups were randomized by standard randomization software by the computer. And serum progesterone level was measured 24 hours after the trigger.
Oocyte retrieval was done 36 hours of Recombinant HCG Trigger and 35 hours after Triptorelin acetate trigger. Oocyte cumulus complexes were cultured in a medium containing hyaluronidase for 1-2 hours. Oocytes were separated from the cumulus complex and the maturity of the oocytes was assessed and graded as "Metaphase 2" oocytes when the Oocyte has visible polar body and "Metaphase 1" when there was no visible Polar body and no germinal vesicle in the ooplasm and "Germinal vesicle" when the germinal vesicle was seen (Figure 1a-c).

\section{Result and analysis}

The groups were analyzed by age, body mass index (BMI), antral follicular count (AFC), anti mullerian hormone (AMH) level and serum estradiol value on the day of trigger.

\section{Age}

The mean value was $30.24+\mid-3.23$ in the Recombinant HCG trigger group and $29.17+\mid-3.15$ in the Triptorelin acetate trigger group. The $p$ value was 0.0858 . Mentioned in table 1 .

AMH: The mean value was $2.21+\mid-0.60$ in the Recombinant HCG trigger group and $3.02+\mid-0.7$ in Triptorelin acetate trigger group. The $p$ value was $<0.0001$.

BMI: Mean value was $24.70+\mid-2.86$ in Recombinant HCG trigger group and $26.52+\mid-2.06$ in the Triptorelin acetate trigger group. The $p$ value was 0.0003 .

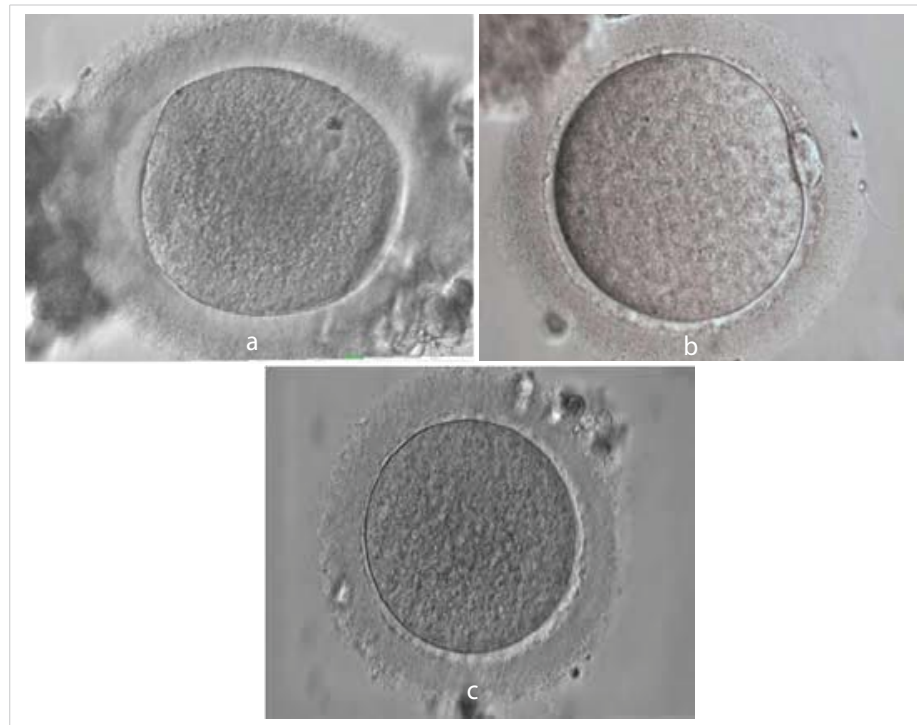

Figure 1: Stages of oocytes maturation. a: Germinal vesicle oocyte. b: Metaphase I Oocytes. c: Metaphase II Oocytes.

\begin{tabular}{|c|c|c|c|}
\hline Table 1: Recombinant HCG \& Triptorelin acetate trigger according to variables. \\
\hline Variables & $\begin{array}{c}\text { Recombinant } \\
\text { HCGrigger }\end{array}$ & $\begin{array}{c}\text { Triptorelin acetate } \\
\text { trigger }\end{array}$ & $\boldsymbol{p}$ value \\
\hline AGE & $30.24+\mid-3.23$ & $29.17 \pm 3.15$ & 0.0858 \\
\hline BMI & $24.70+\mid-2.86$ & $26.52+\mid-2.06$ & 0.0003 \\
\hline AMH & $2.21+\mid-0.60$ & $3.02+-0.76$ & $<0.0001$ \\
\hline AFC & $15.20+\mid-4.51$ & $19.06+\mid-4.44$ & $<0.0001$ \\
\hline E2 & $3427.24+\mid-344.26$ & $3773.02+\mid-591.80$ & 0.0003 \\
\hline
\end{tabular}




\begin{tabular}{|c|c|c|c|}
\hline Characteristics & Recombinant HCG Trigger & Triptorelin Acetate Trigger & $p$ value \\
\hline Total Dose of Gonadotropin (IU) & $3029.94+\mid-1020.17$ & $3269.19+\mid-730.17$ & 0.1667 \\
\hline Duration of stimulation (Days) & $11.61+\mid-0.83$ & $11.68+\mid-0.95$ & 0.6855 \\
\hline Lead follicular size (mm) & $24.19+\mid-0.44$ & $23.80+\mid-1.62$ & 0.0909 \\
\hline Number of Follicles larger than $10 \mathrm{~mm}$ & $13.33+\mid-4.73$ & $20.28+\mid-5.31$ & $<0.0001$ \\
\hline Number of follicles aspirated & $12.33+\mid-3.93$ & $18.85+\mid-5.20$ & $<0.0001$ \\
\hline Proportion of follicles aspirated compared to expected & $92.82+\mid-11.69$ & $91.28+\mid-14.16$ & 0.5406 \\
\hline M2 oocytes & $9.11+\mid-3.85$ & $14.26+\mid-4.73$ & $<0.0001$ \\
\hline Proportion of M2 oocytes compared to total retrieved oocytes \% & $72.86+\mid-17.66$ & $74.89+\mid-15.06$ & 0.5241 \\
\hline M1 oocytes & $1.87+\mid-1.41$ & $2.70+\mid-1.97$ & 0.0136 \\
\hline Proportion of M1 oocytes compared to total retrieved oocytes \% & $16.49+\mid-15.03$ & $13.47+\mid-9.31$ & 0.2153 \\
\hline GV oocytes & $1.61+\mid-0.95$ & $1.94+\mid-1.45$ & 0.1660 \\
\hline Proportion of GV oocytes compared with total retrieved oocytes \% & $13.77+\mid-7.64$ & $10.37+\mid-7.16$ & 0.0194 \\
\hline
\end{tabular}

AFC: The mean value was $15.20+\mid-4.51$ in the Recombinant HCG trigger group and $19.06+\mid-4.44$ in the Triptorelin acetate trigger group. The $p$ value was $<0.0001$.

S. E2 level: The mean value was $3427.24+\mid-344.26$ in the Recombinant HCG trigger group and $3773.02+\mid-591.81$ in the Triptorelin acetate trigger group. The $p$ value was 0.0003 .

Total dose of gonadotropin: The mean value was in recombinant Human chorionic gonadotropin and in Triptorelin acetate trigger group. $p$ value was 0.1667 .

Duration of stimulation: The mean value was in recombinant Human chorionic gonadotropin and in Triptorelin acetate trigger group. $p$ value was 0.6855 .

Lead follicular size: The mean value was in recombinant Human chorionic gonadotropin and in Triptorelin acetate trigger group. $p$ value was 0.0909 .

Number of follicles larger than $10 \mathrm{~mm}$.

The mean value was in recombinant Human chorionic gonadotropin and in Triptorelin acetate trigger group. $p$ value was $<0.0001$.

Number of follicles aspirated.

The mean value was in recombinant Human chorionic gonadotropin and in Triptorelin acetate trigger group. $p$ value was $<0.0001$

Number of follicles aspirated compared to expected.

The mean value was in recombinant Human chorionic gonadotropin and in Triptorelin acetate trigger group. $p$ value was 0.5406 .

Number of M2 oocytes retrieved (Oocyte maturity rate).

The mean value was in recombinant Human chorionic gonadotropin and in Triptorelin acetate trigger group. $p$ value was $<0.001$.

Proportion of M2 oocytes compared to total retrieved oocytes.
The mean value was in recombinant Human chorionic gonadotropin and in Triptorelin acetate trigger group. $p$ value was 0.5241 .

Number of M1 oocytes.

The mean value was in recombinant Human chorionic gonadotropin and in Triptorelin acetate trigger group. $p$ value was 0.0136 .

Proportion of M1 oocytes compared to total retrieved oocytes.

The mean value was in recombinant Human chorionic gonadotropin and in Triptorelin acetate trigger group. $p$ value was 0.2153 .

Number of GV oocytes.

The mean value was in recombinant Human chorionic gonadotropin and in Triptorelin acetate trigger group. $p$ value was 0.1660 .

Proportion of GV oocytes compared with total retrieved oocytes.

The mean value was in recombinant Human chorionic gonadotropin and in Triptorelin acetate trigger group. $p$ value was 0.0194 .

\section{Discussion}

An adequate knowledge about the endocrine and temporal requirements for the maturation of oocytes enables the optimization of IVF protocols and the development of standards to induce oocyte maturation to improve both the safety and efficacy of IVF treatment.

Few studies showed similar or better Oocyte maturation , with the use of GnRH agonist trigger compared to hCG trigger [7,8]. Unlike hCG trigger, GnRH-a trigger stimulates FSH surge in addition to LH surge. FSH surge, in the midcycle, has a specific effect on oocyte maturation and leads to a further expansion of cumulus cells surrounding the oocyte and release of proteolytic enzymes involved in the process 
of ovulation [9]. Lamb, et al. [10] by adding a dose of FSH to the hCG trigger, showed better recovery of oocyte and higher fertilization rates in IVF compared with hCG trigger alone.

This method also enables more nuclear maturity and the resumption of meiosis and thereby increasing the number of Metaphase II oocytes [11]. Shapiro et al showed that increased levels of LH following injection of hCG was slower than that following GnRH-a trigger [12].

The present prospective randomized study was done to compare the efficacy of GnRH agonist Triptorelin acetate 0.2 mg administered subcutaneously and recombinant HCG 250 mg administered subcutaneously, for triggering final oocyte maturation in antagonist co-treated ICSI cycles. The results show that patients triggered with Triptorelin acetate had more yield of mature metaphase 2 oocytes than the patients triggered with Recombinant HCG. This may be due the fact that administration of $\mathrm{GnRH}$ agonist results in endogenous rise in both LH and FSH levels from the pituitary gland owing to initial flare effect similar to that of natural cycle [13]. Whereas recombinant HCG causes only LH rise. GnRH agonist also causes maturation of oocytes, meiosis reinitiation expansion of cumulus cells and thereby giving more number of mature oocytes $[14,15]$.

\section{Conclusion}

In conclusion both Triptorelin acetate and recombinant HCG triggers are effective for final oocyte maturation. Triptorelin acetate, may yield more number of mature metaphase II oocytes, than the recombinant HCG tigger. Nevertheless, more studies and researches might be needed to confirm our findings and improve our understanding.

\section{References}

1. World Health Organization. Prevalence of moderate and severe disability (in millions), by leading health condition associated with disability, and by age and income status of countries.

2. Shoham Z, Schacter M, Loumaye E, Weissman A, MacNamee M, et al. The luteinizing hormonesurge-the final stage in ovulation induction: modern aspects of ovulation triggering. Fertil Steril.1995; 64: 237-251. PubMed: https://pubmed.ncbi.nlm.nih.gov/7615097/

3. Voronina E, Wessel G. The regulation of oocyte maturation. Curr Top Dev Biol. 2003; 58: 53-110.

PubMed: https://pubmed.ncbi.nIm.nih.gov/14711013/

4. Cheung LP, Lam PM, Lok IH, Chiu TT, Yeung SY, et al. GnRH antagonist versus long $\mathrm{GnRH}$ agonist protocol in poor responders undergoing IVF: a randomized controlled trial. Hum Reprod. 20: 616-621. PubMed: https://pubmed.ncbi.nIm.nih.gov/15608037/
5. Huirne JA, Homburg $\mathrm{R}$ and Lambalk CB. Are $\mathrm{GnRH}$ antagonists comparable to agonists for use in IVF? Hum Reprod. 2007. 22: 2805-2813. PubMed: https://pubmed.ncbi.nlm.nih.gov/17872909/

6. Olivennes F, Belaisch-AllartJ, Emperaire JC, Dechaud H, Alvarez S, etal. Prospective, randomized, controlled study of in vitro fertilizationembryo transfer with a single dose of a luteinizing hormone-releasing hormone (LH-RH) antagonist (cetrorelix) or a depot formula of an LHRH agonist (triptorelin). Fertil Steril. 2000; 73: 314-320. PubMed: https://pubmed.ncbi.nlm.nih.gov/10685535/

7. Humaidan P, Polyzos N, Alsbjerg B, Erb K, Mikkelsen A, et al. GnRHa trigger and individualized luteal phase $\mathrm{hCG}$ support according to ovarian response to stimulation: two prospective randomized controlled multicenter studies in IVF patients. Hum Reprod. 2013; 28: 2511-2521. PubMed: https://www.ncbi.nlm.nih.gov/pmc/articles/PMC5054292/

8. Reddy J, Turan V, Bedoschi G, Moy F, Oktay K. Triggering final oocyte maturation with gonadotropin-releasing hormone agonist ( $\mathrm{GnRHa}$ ) versus human chorionic gonadotropin ( $\mathrm{hCG}$ ) in breast cancer patients undergoing fertility preservation: an extended experience. J Assist Reprod Genet. 2014; 31: 927-932.

PubMed: https://pubmed.ncbi.nlm.nih.gov/24854484/

9. Richards JS, Hernandez-Gonzalez I, Gonzalez-Robayna I, Teuling E, Lo Y, et al. Regulated expression of ADAMTS family members in follicles and cumulus oocyte complexes: evidence for specific and redundant patterns during ovulation. Biol Reprod. 2005; 72: 1241-1255.

10. Lamb JD, Shen S, McCulloch C, Jalalian L, Cedars MI, et al. Folliclestimulating hormone administered at the time of human chorionic gonadotropin trigger improves oocyte developmental competence in in vitro fertilization cycles: a randomized, double-blind, placebocontrolled trial. Fertil Steril. 2011; 95: 1655-1660.

PubMed: https://pubmed.ncbi.nlm.nih.gov/21315341/

11. Andersen $\mathrm{CY}$, Leonardsen L, Ulloa-Aguirre A, Barrios-De-Tomasi J, Moore $\mathrm{L}$, et al. FSH-induced resumption of meiosis in mouse oocytes: effect of different isoforms. Mol Hum Reprod.1999; 5: 726-731.

12. Shapiro BS, Andersen CY. Major drawbacks and additional benefits of agonist trigger-not ovarian hyper stimulation syndrome related. Fertil Steril. 2015; 103: 874-878.

PubMed: https://pubmed.ncbi.nlm.nih.gov/25707333/

13. Gonen Y, Balakier H, Powell W, Casper RF. Use ofgonadotropinreleasing hormone agonist to trigger follicular maturation for in vitro fertilization. J ClinEndocrinol Metab. 1990; 71: 918-922. PubMed: https://pubmed.ncbi.nlm.nih.gov/2119392/

14. Andersen $\mathrm{CY}$, Humaidan P, Ejdrup HB, Bungum L, Grøndahl ML, et al. Hormonal characteristics of follicular fluid from women receiving either $\mathrm{GnRH}$ agonist or hCG for ovulation induction. Hum Reprod. 2006; 21: 2126-2130.

PubMed: https://pubmed.ncbi.nlm.nih.gov/16682403/

15. Humaidan $P$, Westergaard LG, Mikkelsen AL, Fukuda M, Yding Andersen C. Levels of the epidermal growthfactor-like peptide amphiregulin in follicular fluid reflect the mode of triggering ovulation: a comparison between gonadotrophin-releasing hormone agonist and urinary human chorionic gonadotrophin. Fertil Steril. 2011b; 95: 2034-2038. PubMed: https://pubmed.ncbi.nlm.nih.gov/21377153/

16. Mascarenhas MN, Flaxman SR, Boerma T, Vanderpoel S, Stevens GA. National, regional, andglobal trends in infertility prevalence since 1990:a systematic analysis of 277 health surveys. PLoSMed. 2012; 9: e1001356.

PubMed: https://pubmed.ncbi.nlm.nih.gov/23271957/ 